\title{
The Living Room of the Future
}

\author{
Neelima Sailaja, James Colley, Andy Crabtree \\ School of Computer Science, University of Nottingham, UK. \{firstname.lastname\}@nottingham.ac.uk \\ Adrian Gradinar, Paul Coulton \\ Imagination, Lancaster University, UK. \{initial.lastname\}@lancaster.ac.uk \\ Ian Forrester, Lianne Kerlin, Phil Stenton \\ BBC R\&D, Media City, Salford, UK. \{firstname.lastname\}@bbc.co.uk
}

\begin{abstract}
Emergent media services are turning towards the use of audience data to deliver more personalised and immersive experiences. We present the Living Room of The Future (LRoTF), an embodied design fiction built to both showcase future adaptive physically immersive media experiences exploiting the Internet of Things (IoT) and to probe the adoption challenges confronting their uptake in everyday life. Our results show that audiences have a predominantly positive response to the LRoTF but nevertheless entertain significant reservations about adopting adaptive physically immersive media experiences that exploit their personal data. We examine 'user' reasoning to elaborate a spectrum of adoption challenges that confront the uptake of adaptive physically immersive media experiences in everyday life. These challenges include data legibility, privacy concerns and potential dystopias, concerns over agency and control, the social need for customisation, value trade-off and lack of trust.
\end{abstract}

\section{CCS CONCEPTS}

- Ubiquitous and Mobile Devices • Interaction Design Process and Methods • Empirical Studies in HCI

\section{KEYWORDS}

Object-Based Media; Internet of Things; Personal Data; Adaptive Physically Immersive Media; Adoption Challenges.

\section{Introduction}

Emergent media services are increasingly data-driven in a bid to deliver highly personalised experiences. The introduction of Object-Based Broadcast (Shotton et al. 2015) is shifting mainstream media from pre-packaged single stream broadcasts to broadcasts having multiple narrative pathways tailored to the viewer's context. This opens up new possibilities in which personal data is leveraged in media production (BBC 2016) where data is used, for example, to stitch together user generated content from different users at live events (Schofield et al. 2015 ) or to tailor news formats that are personalised on user televisions to provide more personally relevant content (Bentley et al. 2014). The turn to personal data in delivering personalised media experiences goes beyond interactivity, which has seen many iterations and diversifications over the years and where users create or influence a storyline through direct action (e.g., Ursu et al. 2008a \& 2008b, Cesar et al. 2008, Cesar and Chorianopoulos 2009, Hanley et al. 2009, Obrist et al. 2015) to enable adaptive content that exploits contextual information from within the consumer's environment to drive personalisation.

An early example of this turn towards data as a driver of adaptive media content is provided by 'perceptive media' (Gradinar et al. 2015), which exploits sensors and Internet of Things devices to allow for the subtle adaptation of the narrative without any direct interactions from audience members. This approach treats data generated by connected devices as 'diegetic influencers' produced within and reflexively shaping the unfolding narrative. The resulting experience is akin to how a person telling a story might adapt aspects of it to a particular location and cohort, referencing landmarks and places in the local vicinity and making use of specific idioms, expressions, or sayings to ensure the story resonates with the audience. Building upon these early efforts, we instantiate a novel physically immersive media installation - the Living Room of the Future (LRoTF) - in which media content adapts in response to user data collected from IoT devices situated in the living room and the adaptation, in turn, actuates connected devices (e.g., speakers, lights, window blinds, heating, etc.) in order to enable the media experience to reach beyond the screen into the living room.

This paper describes the design of the LRoTF and its deployment and evaluation. The LRoTF is a design fiction (Coulton et al. 2018) intentionally constructed to probe a novel design space that reaches beyond virtual forms of immersion to consider the potential of adaptive forms of media that trade on and exploit personal data generated 
by connected IoT devices to drive highly personalised, physically immersive experiences. It is broadly recognised that while the use of personal data enables service innovation, it also introduces significant adoption challenges due to broad societal concern (Lanier 2014). At the outset the LRoTF thus incorporates a Human Data Interaction (HDI) (Mortier et al. 2016) and Databox approach (Crabtree et al. 2018), which seeks to preserve privacy in the use of personal data and enable end-user agency and control to mitigate critical adoption barriers.

The results of the deployment show that audiences found the LRoTF highly immersive, engaging and 'magical'. However, audience members were clear about the demarcation between enjoying a novel media experience versus adopting it in their everyday lives, which we unpack in explicating the deployment study findings. The findings articulate a spectrum of adoption challenges (Lindley et al. 2017) confronting physically immersive media: not only privacy, but a range of concerns including data legibility, potential dystopias, agency and control issues, the social need for customisation, asymmetrical value trade-off, and lack of trust that confront the uptake of new physically immersive media experiences in everyday life, which media research and design needs to be sensitive to when moving beyond virtual immersion into the living room of the future.

\section{Design of the Living Room of The Future}

Immersive media experiences are largely virtual and 'liminal' in nature, insofar as they move the audience between states (from reality to virtual reality). However, this approach can work against immersion as it may induce cognitive dissonance if the audience experiences significant differences between those states. This is particularly noticeable in virtual reality, where the audience's visual perception is not matched with their other physical senses, causing adverse physical reactions such as nausea (Regan 1995). An alternate approach, and one adopted in creating the LRoTF, is to consider immersion from a 'liminoid' perspective (Coulton 2017) and the creation of a hybrid reality that dynamically adapts components of a media narrative in response to the audience's physical context through the use of connected 'Internet of Things' (IoT) devices.

Current IoT applications are predominantly focused on smart-homes and large-scale sensing. However, the possibility that IoT presents to media research, particularly of understanding and responding to audience contexts on an ubiquitous scale, makes it an effective resource for creating an intelligent sensing environment for the LRoTF. Early explorations of this approach are provided by 'perceptive media' (Gradinar et al. 2015) and latterly by the 'IoT storytelling' project (Coulton 2015), in which narrative was contextualised for the audience by augmenting broadcast media with a number of IoT objects including lights, a connected doll, and an olfactory device, which were synchronised to particular on-screen events within a BBC documentary to create a broadcast experience which utilised household objects within the viewer's own context.

The LRoTF extends this approach to incorporate IoT devices as both sensors that collect real-time data from the audience environment that drive media adaptation and as actuators that respond to specific media adaptations to create highly personalised, bi-directional, physically immersive media experiences. The LRoTF embeds this approach in an embodied design fiction (Coulton et al. 2018) that incorporates both real and fictional technologies in a mundanely familiar environment that nevertheless allows people to get their hands on the future and experience what it might look and feel like for themselves. This 'mundane experience' approach, in turn, enables people to reflect on what place future technology might have in their everyday lives and to articulate challenges that confront its adoption.

\subsection{Key Design Components}

Key design components of the LRoTF include the physical space, the media component, connected objects, and the HDI component, all of which combine to deliver the audience experience.

The Physical Space. The living room represents a familiar and readily identifiable space in which media is consumed in many homes. It is of course not the only space in which media is consumed in the home, but it lends itself well to 'world building' (ibid.) or crafting an embodied experience that may be situated in public spaces to facilitate broad public engagement. To support deployment, the LRoTF is intentionally small (approx. 3.5 metre square). Nonetheless it houses furniture one might find in a typical living room: a sofa, coffee table, side tables, lamps, and a TV. 


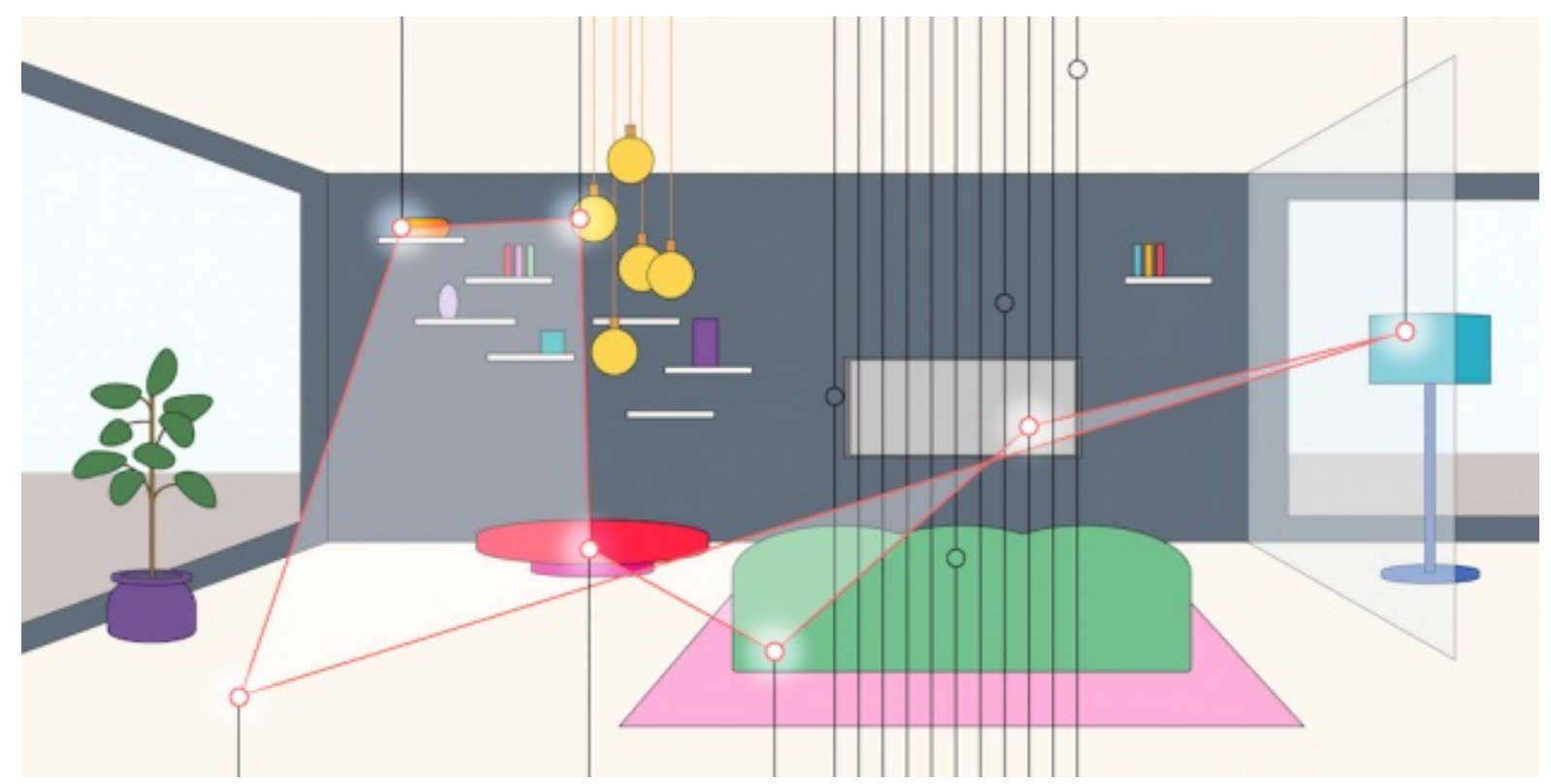

Figure 1. Initial sketch of the LRoTF.

The Media Component. Media content for the LRoTF was developed by two successful candidates - Ilihja Tiricovski and Pavel Prokopic - selected from a workshop held as part of the British Council's 'PlayUK' programme at the regional innovation forum Sarajevo Unlimited (British Council 2018). Details of the workshop and subsequent media development are beyond the scope of this paper, suffice to say that the final output consisted of a set of 'object based media' (Smith 2018) rendering two different films in a split screen format. Multiple pathways could be taken through either film, changing both the content and the order in which parts are played, in response to the data generated by connected objects in the LRoTF.

Connected Objects. The LRoTF exploited a mix of off-the-shelf and bespoke connected objects which include connected lamps, a high-end fan blowing hot as well as cold air, a window blind controller, and a bespoke clock radio that acts as an audio speaker to play ambient sounds. Alongside these objects, which can be considered as 'outputs', in that they are controlled by media objects, we also added a number of data 'inputs' that directly affected the media content. These consisted of pressure sensors and NFC readers. The pressure sensors were embedded within the sofa to detect when the audience sat down, and NFC readers were integrated into the tables to detect when drinks and magazines were interacted with by audience members.

Human Data Interaction (HDI). The LRoTF enables interaction between object based media and connected objects via the Databox platform to dynamically contextualise media objects using data generated by viewers' interactions with connected objects in the living room. To surface the fact that the LRoTF experience was driven by data generated through audience members' interactions with connected objects, we situated a small tablet on the coffee table to act as a data monitor. The monitor displays a scrolling textual list of 'data announcements' to make it visible that data is being collected and used to trigger filmic events and personalise the experience, though not all uses of the data were real, e.g., 'emotional analysis' was a fictional element of the experience.

The LRoTF Experience. The above components combined to enable the LRoTF experience, which was designed to last between five and ten minutes depending on audience interactions with connected objects. The experience begins when the audience sits on the sofa: the pressure sensors in the seat cushions trigger the window blinds to close automatically, the lights to adjust their colour, and the movie to begin. The 'voice of the living room', which comes from the clock radio, introduces audience members to the LRoTF. The lights adjust colour and brightness to ambiently match the on-screen content as the film unfolds. When the wind audibly blows through trees or a girl's hair in the film, the fan blows warm air onto the audience. When an audience member engages with a smart object, the data collected is displayed on the monitor and the film adapts in real time to these serendipitous interactions to create a unique 'cut' through. If an audience member picks up a book from the magazine rack about the particular building in Sarajevo in which the film was partially shot, for example, then extra footage is added about the building to the experience; picking up a travel card adds more film of an underground train journey; picking up a drinks bottle adds more shots involving rain; and picking up a TV remote control triggers the voice of the living room to say 'Do you think the living room of the future can be controlled with a remote?' After the film has finished, a UV light is initialised to highlight connected objects, data flows and the Databox, all of which use fluorescent materials in their construction to enable this ' $U V$ reveal'. The voice of the living room then invites the audience to experiment with the connected objects and view an alternative version of the film driven by 
conscious exploration (rather than serendipity). At the end of the session the lights come on, the window blind is raised, and a receipt detailing the data generated by the audience to drive their experience is provided by a thermal printer built into the coffee table. The receipt highlights what items have been interacted with and their effect on the path taken through the film. It also highlights that all the data has been processed securely within the Databox and not passed to external parties. ${ }^{1}$

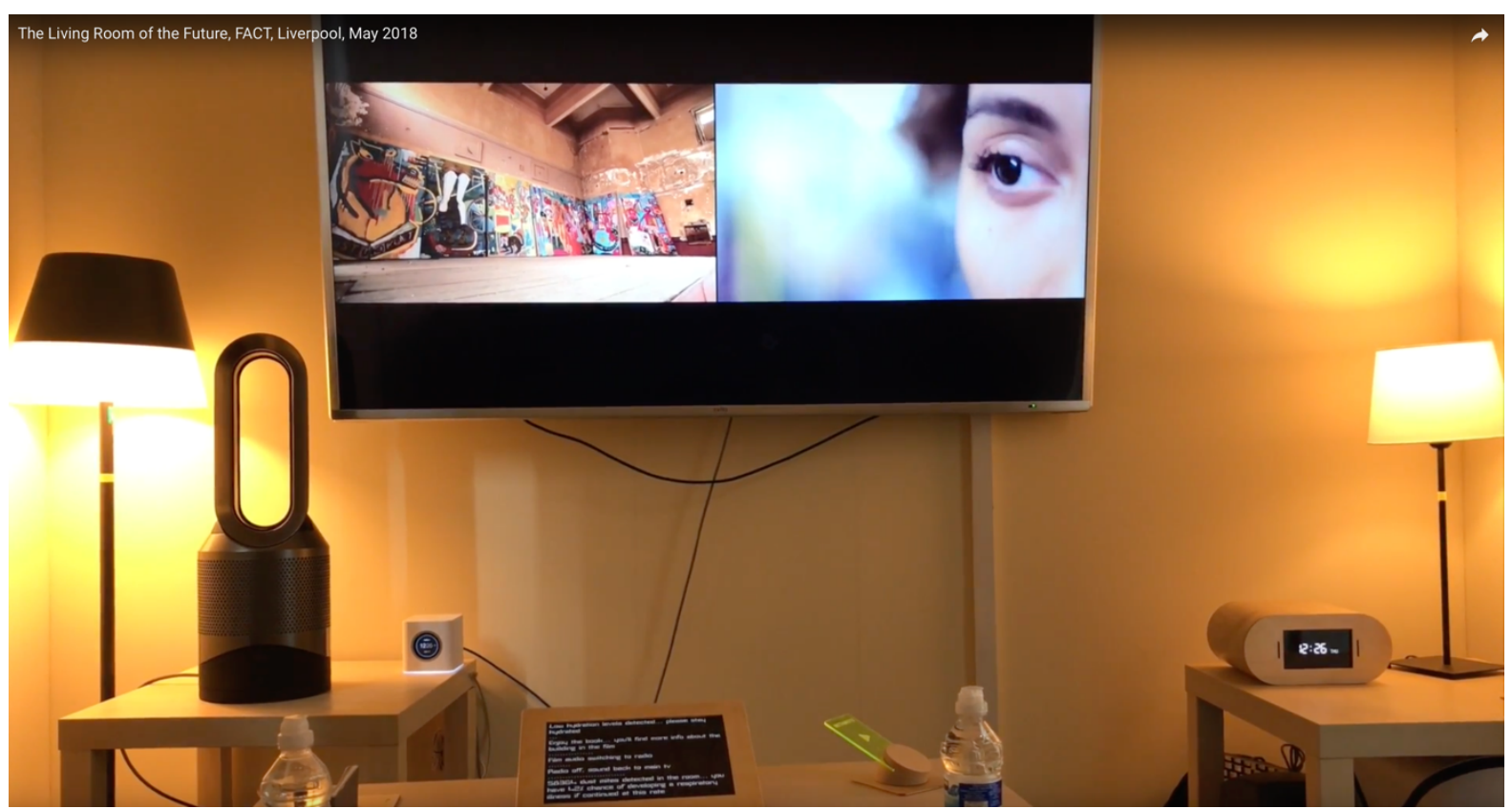

Figure 2. The LRoTF - an embodied design fiction for probing the future.

\section{Evaluating the LRoTF}

The LRoTF was evaluated using an 'in the wild' approach, which seeks to elicit naturally occurring responses to novel technologies in contrast to the carefully crafted responses that result from highly controlled lab-based experiments (Crabtree et al. 2013, Rogers and Marshall 2017). The LRoTF was thus deployed, and made available to the public at large, at the Foundation for Art and Creative Technologies (FACT) in Liverpool for an initial planned period from May 4 to May 8, 2018. However, response to the LRoTF was such that the deployment was extended for another 15 days, during which time more than 2000 people visited the LRoTF. The experience has since been deployed at the British Council's Play Festival in Skopje, Macedonia, and at the V\&A as part of its Digital Design Weekend. Nonetheless, our evaluation focuses here on the initial planned period of deployment at FACT, during which time 59 people in 30 groups of up to 3 agreed to take part in our study.

The groups were a mixed bag of people who walked in off the streets, some of whom knew each other and others who were strangers. No reward was offered for participation, and most had not turned up to specifically experience the LRoTF, rather the majority were simply spending a day or afternoon 'in town' and 'taking a look' around FACT in the course of doing so. Before entering the LRoTF, participants in the study completed ethics documents, approved by the University of Nottingham's Computer Science Ethics Committee, and a short demographic survey. All participants were above the age of 18. The cohort consisted of 26 females and 33 males from diverse ethnicities (White British, White Irish, Asian Indian, Asian Chinese, Middle Eastern, Mixed) and nationalities (English, Irish, Chinese, Indian, Romanian, Cypriots, Macedonian etc.). While we make no claims to the representativeness of the cohort, participants had a similarly diverse array of occupations, ranging from barmen, nurses, students, film makers, producers, software developers and designers to landscape architects, account executives, animators, marketing specialists, gardeners, journalists, researchers, corporate managers, company directors and civil servants. 36 participants reported that they had heard about the Internet of Things and 51 said they owned at least one connected device. 43 participants reported that they were 'concerned' about data privacy, 12 responded 'maybe', and 3 said 'no'.

\footnotetext{
${ }^{1}$ A video of the LRoTF is available here: https://www.youtube.com/watch?time continue=2\&v=yneprnGCRm0

2 The apparent 'randomness' of our sample was raised in discussion of this paper. As noted above, in-the-wild research contrast sharply with highly controlled lab-based experiments. The real world consists of heterogenous
} 
After the experience, the participants took part in an exit interview, which was recorded on audio and subsequently transcribed. The data receipt and a schematic diagram of the connected objects and data flows within the LRoTF were used to seed conversation and probe participants' experience. The interviews were informal and unstructured, driven by what participants had to say about the LRoTF rather than a pre-planned script and series of questions. We sought to root discussion of the LRoTF and the innovation it represents both in the participants' immediate personal experience and their mundane reasoning about the projected role and place of such services in their everyday lives. We thus focused discussion on 3 broad areas of interest: participants' immediate response to the LRoTF experience; their response to the collection and use of personal data to drive such experiences; and their reasoning about future physically immersive, data-driven media experiences in their own everyday lives.

Subsequent analysis of the interview transcripts attended to the 'endogenous topics' (Pollner 2010) attended to and elaborated by participants and the interviewers in their talk together. Our analysis is thus framed around vignettes or conversational extracts that display the distinctive topics that populated discussion of the LRoTF, rather than external and/or a priori codifications. In short, our analysis thus attends to how our participants accounted for the LRoTF in responding to our broad areas of interests:

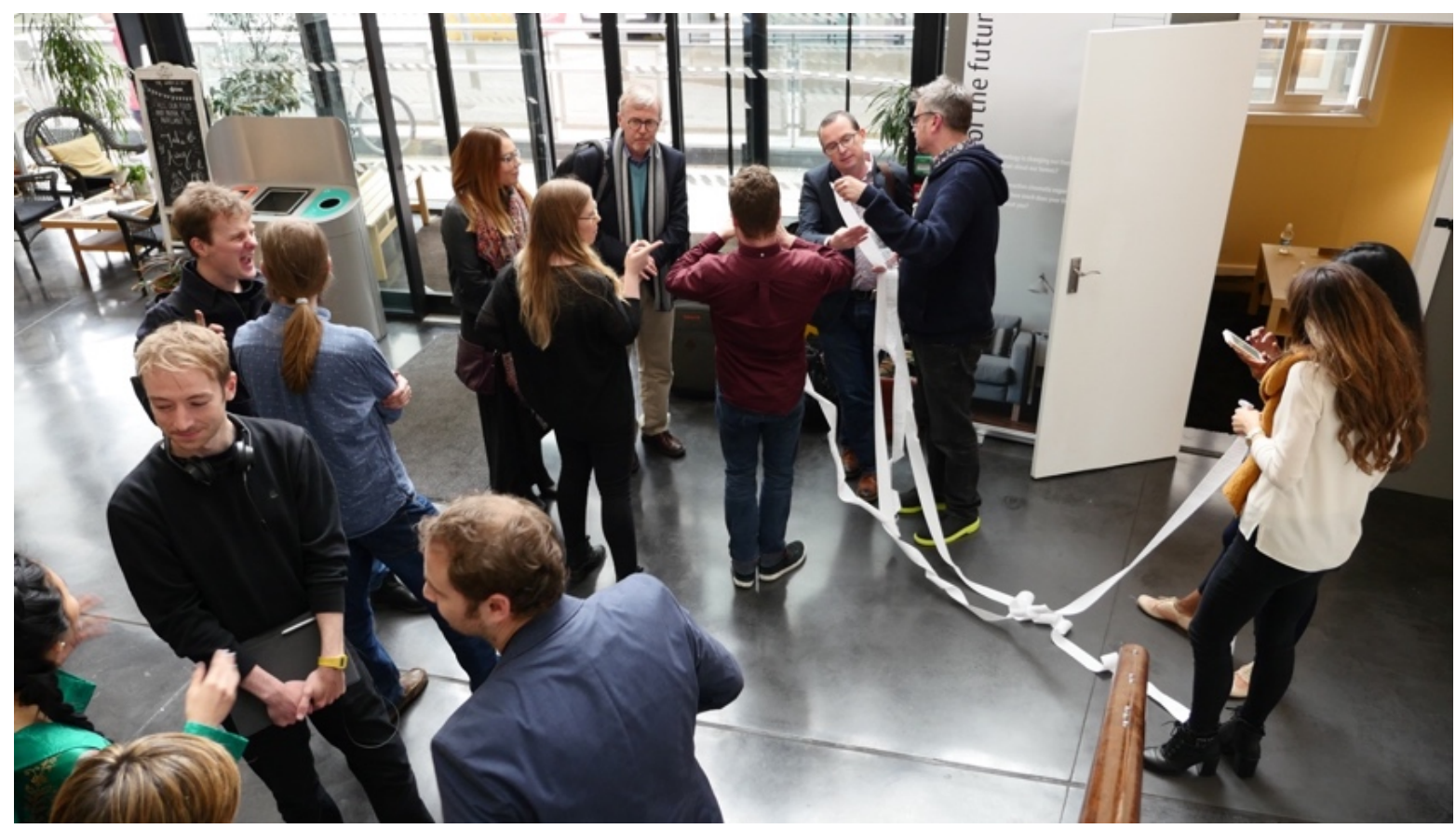

Figure 3. The LRoTF at FACT.

Clayton: It was really good. It was a different experience. Like, it was a bit mad, but in a good way. I don't know, I wasn't expecting it to be like that. It wasn't half a bit intense. Like, I don't know, I want my living room to be like that.

Interviewer: Oh, do you?

Clayton: Yes. Like, that was really cool. Like, the lights changing and everything according to what was going on on the TV, and then the fan coming on and off and stuff. It was 'wow'! Like, I don't know, it just messed with my mind ... really cool. This has made my day a really good day now.

Alex: To my friends l'd use the word 'magic'.

Interviewer: Magic?

Alex: I would say it's magic. It's just, it's in sync. It just happens with what you're doing, and whoever's in the room controls it.

Sarah: It was alive. The lights changing, anything I moved was reacting, it was a heartbeat, that room.

Clayton, Alex and Sarah were and are in no way special cases; all of our respondents found the LRoTF thoughtprovoking and engaging. Participants were particularly receptive to the synergy between physical objects and media content and reported that the interplay between the two made for a richer, more 'sensory' experience:

cohorts, a small slice of which is included here. Furthermore, we are not interested in analysing the LRoTF by cohort characteristics (age, gender, occupation, etc.) to furnish statistical insights, but lived experience and the challenges this raises for design. 
Jo: It was good. I didn't expect it to be as sensory. I thought there would be an interconnectivity of all the objects in some manner, but say if there was a yellow hue on the screen, that would then reflect in the lighting, which I thought was quite nice.

Lee: The way the room was reflecting what you were seeing colourwise - if there were shots where there was a lot of greenery on it then the room was turning green. Then there was a lemon, it was turning lemon. It just seemed to reflect what you were looking at really.

Jon: Also, like, the breeze. Like on the train, the underground.

Lee: I liked that moment. Her hair was blowing and then suddenly the fan went on. It was nice.

Jo: And the temperature, yes. Was that just coincidence that it linked in with the train scene?

Respondents generally reported high levels of immersion, which they attributed to the LRoTF 'reacting' to them and making them feel that they were 'in the film':

Alice: It was very immersive.

Dave: It gives you a better feeling of watching a movie.

Alice: Yes.

John: It just makes the whole experience more enjoyable. It's not just looking at a 2D screen in front of you. It's creating the whole mood and everything.

Dave: It makes it more exciting.

Alice: Yes, it could benefit a lot of films, giving you that experience of being the star of the film, in the film, kind of thing.

The overall response to the LRoTF was largely positive: mad, intense, alive, magical, reacting to the viewer and immersing them in a rich sensory media experience. However, that is not to say that the LRoTF is without its problems. Respondents had a number of reservations regarding the collection and use of personal data and the prospect of living with data-driven media experiences in their everyday lives, both of which impact the potential for adoption and are considered in turn below.

\section{Reflections on the Collection and Use of Personal Data}

The LRoTF trades on the collection and use of personal data generated by IoT devices, which we intentionally surfaced through the data monitor, UV reveal and data receipt. These design decisions allowed us to probe audience member's reactions to the collection and use of personal data and to engage them in discussions articulating their concerns and expectations regarding data-driven media services in everyday life. Many respondents acknowledged the potential benefits of enabling service providers to access their data and were happy to do so if they saw value in the process, but not without reservation or caveat. Our respondents' willingness to 'share' data was conditional, depending on a number of factors that are key to ensuring acceptable data collection and usage practices in the intelligent, self-thinking media environments of the future:

Interviewer: Did you understand that the experience was driven by your interactions and data?

Martin: The small screen in front of us was showing what was going on, even down to saying what elements are in the room in terms of dust and things like that and how it could affect you.

Lesley: As soon as I realised I was more focused on the fact that everything was actually data-driven and the whole idea that data is subliminally being taken from us all the time became kind of more apparent I suppose.

Interviewer: At what point did it become apparent?

Lesley: I think immediately when you took the book out and the screen said 'enjoy the book', and I was like, 'right, OK' and I picked up the travel card and it was like, 'OK yes.' It's like a stripped down way of how in everyday life those bits of data are always collected from you. Like I say, the travel card's a really good example. You use it every day and you never think about it, when really it's collecting a lot of data about where you are, how much money you spend, where you're going, what times you're going. You're forever, kind of, getting data collected.

Interviewer: If this could become your living room in the near future, would you like to know about the data that flows in the background?

Arianna: l'd definitely want to know.

Interviewer: Why?

Arianna: I think partly curiosity but also it's a good way to keep track of it, make sure it's not going too far the wrong way.

Respondents generally saw the monitor as a key part of the LRoTF, a source of interaction, communication and feedback between the experience and the audience, which they often looked at in order to try and work out what was happening within the room and in doing so came to see and subsequently voice retrospectively concerns about 'background flows of data' and the need to 'keep track' of them.

In the course of the experience the data monitor encouraged participants to actively engage with the connected objects driving the experience: 
Jack: I just grabbed everything.

Interviewer: You grabbed everything. Did you know that something was happening when you grabbed them?

Jack: I got a response from the small screen on the table, yes.

Interviewer: Did that help you in any way?

Jack: Yes, of course, that was my response, that I interacted with the room and that the room reacted back to me.

Louise: It helped me understand things that I would otherwise not know was happening, like the emotional analysis. I feel if you're doing stuff like that it has to be clear that that's happening and the screen was doing that. If the screen wasn't there then I would have no idea that that sort of data would be collected on me.

In reflection, as Louise makes perspicuous, the data monitor surfaced the need people have to be 'clear about what's happening' when data is being collected, particularly what use it is being put to, e.g., emotional analysis.

The data monitor clearly had distinct affordances and invited participants to probe and engage further with the experience as it unfolded, but it was not without its troubles. Participants felt that there was something important missing from the data announcements:

Paula: The monitor kind of showed what data was being collected, but it didn't show how it was being used.

So, actually, I think because it was like a novel experience, I kind of spent a lot of my time trying to figure out,

'I did this, so now what's happening', rather than focusing on the changes and content.

Stuart: I wasn't quite sure where exactly the connection was. I was aware because the black tablet was telling me like, 'You're doing this and you're doing this.' That was the thing where I could connect. So, some things are changing in here because of me, but I didn't really know how the rest was changing because of me.

In reflection, it became clear that participants' need to understand 'what's happening' with their data extends to include the 'connections' between data and effect so that they can understand how or on what basis particular effects are produced (a point which also surfaced in respect to the data receipt and the choice of film endings).

The gap between the data and its connection to and impact on the experience caused some participants to be 'confused' about what exactly was happening in the LRoTF and why.

Simon: I think, when the book was picked up, some lights overhead lit up so you could read it, but it didn't replicate so l'm not really sure.

The source of the confusion was in part due to design choices made in constructing the LRoTF - we consciously designed it so the LRoTF would not behave like a giant remote control for the TV (so picking a book up or water bottle up again will not 'replicate' an effect as the effect has already been triggered) - but also due to any clear means of controlling what was happening:

George: I was really surprised there wasn't like a Google Home or something in there.

Ahmed: Yes, voice. Frictionless interactions, more humanistic, humanistic to a point where it doesn't feel too futuristic.

Amida: For example, when you pick up the remote, we get a little message: 'Do you really think the living room of the future will be controlled with a remote?' How will it be controlled then, because there wasn't really any way of control? So, I think voice would be a good option.

Our respondents' reflections on the collection and use of personal data as part and parcel of the LRoTF experience highlights several concerns that coalesce around the legibility of data collection and use - keeping track of background data flows, being clear about what's happening with data and the purpose it is being put to, making the connection between data and effect perspicuous - and being able to exercise control over the experience. We do not suggest that an improved data monitor, UV reveal and data receipt will address these concerns in the future, these are provocational devices not solutions, but rather that they should be taken into explicit account when designing data-driven media experiences.

\section{$5 \quad$ Living with Physically Immersive Data-driven Experiences}

When asked to consider the prospect of living with physically immersive data-driven media experiences in their own homes, our respondents' reaction shifted from being positive to a more reserved and indeed reticent stance, articulating further barriers to the adoption of data-driven media experiences in everyday life.

\subsection{Privacy Concerns and Potential Dystopias}

Unsurprisingly the potential use of personal data from connected objects situated within the home provoked privacy concerns. Participants viewed the home as a particularly sensitive context and were wary that data-driven media services would make it possible for external parties to 'snoop' on their intimate habits: 
Peter: If it's printed just for yourself I would be okay with it but if, you know, it's streamed somewhere then it would be more like ...

Xavier: You might change your behaviour.

Peter: Yes.

Xavier: For example, if someone tracked how many times you opened the bottle of wine or a pint of beer and its broadcast, people would probably change their behaviour.

Coupled to the 'streaming' of insights into what goes on in the home, and the potential disruptive effects of one's domestic behaviour, is a concern that knowledge about habits and lifestyles might well be used for purposes outof-context of the media experience, which could also have negative impact on the household members' lives:

Kevin: It's like a health insurance firm looking at it and going, 'Right OK, well if you come for a premium with us we're going to hike the premium up because we know that you are living in a dusty home.' It's one of those.

Respondents posited various dystopian scenarios that mitigated against the uptake of data-driven media experiences in everyday life due to what was seen as their fundamental potential to breach consumer privacy:

Jenny: I feel very shocked because this is my room and I don't want my data to be collected and analysed by another person - there's no privacy! People need some space to live, they need to not be disrupted or something else.

Ramsey: You feel like you're being watched or tracked, like you're not necessarily alone in this room. It's like, 'Are they going to use this for other things, not just for my own home'?

The potentially dystopian effects of streaming personal data and sharing or even brokering it with other parties is a major barrier to the adoption of data-driven media services in everyday life. Concrete assurances will be required of service providers if there is to be any prospect of their future uptake at scale.

\subsection{Agency Concerns}

Respondents expressed significant concerns regarding the impact of data-driven media experiences on their autonomy if they were to find their way into their homes. In addition to the lack of direct control over the media experience itself, as highlighted in section 4, respondents voiced concerns over their ability to control the use of personal data and the impact of autonomous services on their everyday lives. Living in an environment that automatically responds to its inhabitants interactions was seen as something that could potentially undermine one's personal freedom:

Jake: I liked the idea of it, but as I said it takes away your autonomy. When you get bored, you change the channel at the moment, whereas that system might change it for you, and open the blinds, close the blinds, etc. It feels like if I'm not following exactly what's on the screen, then am I doing something wrong. It's a bit like it's telling you how to live your life.

Karl: It felt a bit robotic, because whatever we were doing, the screen was telling us, like, 'Now you're feeling thirsty, have a drink' ... It felt like we were not in control of the environment.

Lynn: My living room, to me, is where I want to go to get away from it all. I don't want to necessarily be prompted to do things.

One potential solution, which runs counter to current enterprise models of personal data use, was seen by respondents to lie in the ability to personally control not only the experience but the data that drives it:

Daz: I want to be able to own that data and negotiate that with them; if you like, be able to pick and choose.

Like Daz, many respondents were sensitive to ownership of their data and having the ability to control who can access it, to be able to 'pick and choose' as it were. At the same time respondents recognised that actively controlling their data could be time-consuming. The issue of data control became a double-edged sword where the need for control was and is perceived as necessary, but the time and effort required becomes a barrier to its proper execution. Respondents were, however, enthusiastic about the way in which the LRoTF handled their data:

William: I don't mind because it deletes it. I like the ethical data thing because something I do worry about is the way our data is used generally. So I think if it was in my own home and I had the security that it would only be used there or deleted, then I would quite like it.

Being able to exercise control over one's environment and what happens in the living room of the future is a major barrier to the adoption of data-driven media services in everyday life. Mechanisms enabling consumers to control media experiences and who can access the data that drives them are needed to foster their uptake at scale. Solutions that only use data locally and delete the data from the service after use hold particular promise. 


\subsection{The Social Need for Customisation}

The need to enable end-user control extends to the need to customise data-driven media experiences around the social circumstances of their consumption:

Sara: So it knows I drank water because I picked up the water bottle. No, it's a bit scary in a way that there's technology that can track what you're doing.

Lily: I felt that there were sensors in the cushions, kind of round - tell me if it's wrong - I had this feeling, and I thought about taking a seat on the ground because the couch suddenly became very suspicious.

Julie: I think that if I went into a neighbour's room or someone who lives in a different city and they were collecting data on me I wouldn't like it, because it's not my personal data that would be needed. I'm not the one that's been targeted, I'm a bystander.

Whether it is a matter of turning sensor data off to avoid 'scaring' people or making participants feel uncomfortable or some other data sources to exclude 'bystanders' from the experience, our respondents felt it important that they be able to tailor the physical context of data-driven media experiences around the social context. This also included optimising media experiences for different members of the cohort:

Terry: Like, if there are three of us, you could at some point know that, 'This is person $A$, this is person $B$, this is person C, and I know that person A has got this problem, and person B may have this problem.' It may adjust things accordingly, but then again, it may have to make a collective decision if the three of you are in at the same time, 'Well, this person's got this problem,' or 'This person's got this problem, this person doesn't.' How do you make it so that it is a good experience for all three at the same time?"

Control is not only about who can access the data then, but also of configuring particular data sources as part of an experience, whether to alleviate the concerns of participants or to enhance the experience for others. A one size fits all model is a significant barrier to the adoption of data-driven media experiences in everyday life.

\subsection{Value trade-off}

Unsurprisingly respondents generally expected that they will receive something in return for the use of the personal data:

Catherine: I'm a, 'here's my data, show me what you can do with it,' kind of person. I'll happily give up personal data if you can give me something in return.

The question of course is what constitutes a 'return'? Some respondents thought 'experience enhancement' enabled through more intelligent media services that go beyond current data-driven media recommendation systems 'valuable'. Others wanted more 'utility' and 'convenience' and envisaged scenarios where the LRoTF shifted to the kitchen and became a 'digital maître d' enabling an immersive cooking experience that helps by automatically turning the stove on/off and up/down, and notifying the user of ingredients that they might be allergic to, etc. Nonetheless, the pre-eminent threat to privacy, and the potentially dystopian consequences that accompany its breaching, diminish the perceived value of data-driven media services for many.

Jack: I'm not sure you get back what you put in. As long as it's kept in that isolated environment [the LRoTF], that's OK, but if it goes out, which I think it inevitably will do, then do you really get back all the services that you want versus all the information you give out? I'm not sure.

Like Jack, many respondents questioned whether the value they get in return for their data is symmetric to the value of the data collected from them to drive innovative media experiences. Irrespective of what form value takes for particular people it would seem that enabling a more symmetrical relationship that demonstrably benefits those whose data is exploited is an important challenge to the widespread adoption of data-driven media experiences in everyday life.

\subsection{Trust}

The value trade-off and uncertainty that accompanies the use of personal data inevitably bleeds into matters of trust. Respondents' reasoning here, as elsewhere in our interviews, was informed by a general concern with the ways in which personal data is currently being exploited in society at large and the scandals that accompany it. This 'data zeitgeist' was further exacerbated by the LRoTF and the hyper-personalisation of media experiences:

Tanya: My trust in broadcast comes from the fact that everyone gets the same as me. That's where my trust fundamentally lies, knowing that everyone else has the same content. So, there's something about this idea that there's some clever processing of data that you're not quite privy to and don't quite know why decisions are being made that might change or alter that experience in some way. I think, for me, there is a risk that that could undermine the trust in the content that I get. 
While they could see benefits in personalisation, our respondents also felt that allowing media experiences to be hyper-personalised could have negative consequences that undermine their trust. Of particular concern was the potential impact on consumer behaviour in 'changing' or 'altering' media experiences in order to deliver highly personalised content:

Adrian: If it would be to influence you in some way or other it would be, like, unethical.

The ethics of what is done with data and the potential to manipulate or 'influence' persons attitudes, behaviour and choices is a core challenge to the adoption of data-driven media services in everyday life. As Tanya hints at, enabling trust very much turns upon transparency in data processing and automated decision-making.

It is also that case that our respondents located trust in the service provider organisation, but 'track record' will not guarantee the acceptability of data-driven media services alone.

Phil: I suppose it would depend for me what company was producing the things, and the track record of the company.

Lenny: Personally, that worries me. I mean people like the BBC, as one example, I trust more than let's say, Fox News or whatever, but it's a future that worries me.

Interviewer: Why does it worry you?

Lenny: I think because it's such new territory. I worry that people aren't thinking enough about the ethics, it's like people moving too fast to really think about the ethics behind the stuff.

Trust in the use of personal data by service providers generally is precarious, even those with good reputations, an article of faith for many respondents that stands on unsafe grounds:

Suzanne: We all put our trust in them, we're kind of like, 'Oh yes, it'll be fine, they won't do anything with my data, everything will be fine.'

Lewis: But it's like what I was saying before about Facebook and that. I don't trust it because someone else has got, like, control of all your data and there are so many times where someone can get hacked and then, your data's out there for everyone to see. There's always someone out there that can hack something.

If consumers are to trust data-driven media experiences then service provider organisations need to go beyond issuing privacy policies and put mechanisms in place that enable consumers to have confidence that they have considered the ethics of leveraging personal data and that their data will not be unduly exploited.

\section{Implications for Adoption}

As detailed by Lindley et al. (2017), adoption is emerging as a "material concern" in human-centred design that reaches beyond longstanding interest with the usability of future and emerging technologies:

"User-centred design methods help us to develop prototypes and move toward designs that are validated, efficient, and rewarding to use. However, these studies rarely shift their temporal focus to consider, in any significant detail, what it would mean for a technology to exist beyond its prototypical implementation, in other words how these prototypes might ultimately be adopted."

Adoption is typically treated as "someone else's problem" (ibid.) insofar such considerations occur much later in the design life-cycle, often after technological solutions have been released to the public when it is too late to remedy problems; take the ubiquitous home router for example and its inability to support the mass of users with non-technical expertise (Crabtree et al. 2014). The LRoTF is not only a substantive exploration of physically immersive media but a methodological innovation that exploits a design fiction approach to instantiate a way for designers to explore the adoption challenges of future and emerging technologies early in the design life-cycle. The LRoTF is not a finished product then, but an embodied and experiential provocation that allows potential end-users to see what future technologies might look and feel like when situated in a mundanely familiar setting, and on that basis, from that perspective, to elaborate what they perceive to be challenges to adoption.

Accordingly we have found that a spectrum of adoption challenges confronts the uptake of physically immersive, data-driven media experiences when respondents were asked to reflect on the collection and use of personal data and to consider the prospect of living with such services in their everyday lives. At a high level our evaluation reveals nothing new: people are concerned about privacy, transparency, trust, control, etc., as found by a host of other researchers (e.g. WEF 2011 \& 2014, Rose et al. 2012, McAlone 2015, Kerlin et al. 2016, Moulding 2017, Sailaja 2017 \& 2018). Indeed, these concerns hallmark diverse research into the 'smart home' and have done so since the early days of ubiquitous computing (e.g., Langheinrich 2002).

In an entertainment context, smart home research has come to focus on the living room as a specific site for development over recent years. HCI researchers have focused on multi-screen media to augment TV broadcasts (Brown et al. 2014), for example. More relevant to the LRoTF, RoomAlive (Jones et al. 2014) is an immersive, augmented gaming experience that dynamically adapts content to provide interactive projections - examples 
include a whack-a-mole game where "players physically whack or shoot moles popping out of their floor, walls and couch" within the room - and the Time Home Pub (Huang et al. 2013) that explores how simple physical objects such as a whiskey glass, a table, and an MP3 player can be exploited as interaction devices.

Complementing these kinds of explorations, the use of data in storytelling is a concept that is gaining momentum in media research. For example, Hooke's (2018) work explores the use of data in interactive storytelling and concludes that data usage helps tell more engaging stories and adds significant value to the media experience. It also highlights the potential data creates for exploring developments in adaptive media, which feature non-linear pathways and surfaces the need to establish ethical production practices that consider "complex issues of ethics, privacy and data ownership"; a point also underscored by previous ethnographic research on media consumption (Bernhaupt et al. 2007).

Clearly there are parallels between our findings and others. However, we are not interested in privacy, transparency, trust, control, etc., in general and set an interest in high-level abstraction aside. Instead we seek to understand the specific challenges that confront a novel class of media that exploits personal data to drive highly personalised, physically immersive experiences. Our substantive takeaways are practical rather than more broadly theoretical then, and focus on the particular challenges that need to be addressed if this novel class of media experience is to find its way into everyday life at scale, rather than synergising our result with the broader literature on privacy, trust, etc.

\subsection{Physically immersive media adoption challenges}

Our evaluation results highlight a spectrum of adoption challenges that media design should be sensitive to when creating adaptive physically immersive, data-driven experiences including data legibility, privacy concerns and potential dystopias, concerns over agency and control, the social need for customisation, value trade-off, and lack of trust. Below we consider the specific issues bound up with these challenges and what needs to be done to address them.

- Data legibility. Physically immersive, data-driven experiences should enable consumers to understand the data flows involved, the purposes the data is being collected and used for, and make the connection between data and effect perspicuous.

- Privacy concerns and potential dystopias. Concrete assurances will have to be provided to the consumers of physically immersive, data-driven experiences to circumvent the threat to privacy and potentially disruptive and dystopian impacts created through the distribution of personal data.

- Agency and control. Physically immersive, data-driven experiences need to enable different levels of human control spanning a) the media experience itself, b) access to personal data, and c) the efficient management of data to mitigate perceived threats to autonomy and agency.

- The social need for customisation. Human control must also extend to customisation of the data sources used by physically immersive, data-driven experiences to enable consumers to tailor the experience to the social context of use and/or optimise performance for differential cohorts.

- Value trade-off. The widespread uptake of physically immersive, data-driven experiences turns upon service providers being able to demonstrate to consumers that the relationship between them is symmetrical and of mutual value.

- Trust. The creation of hyper-personalised experiences raises the spectre of manipulation and threatens consumer trust, which cannot be mitigated by track record and reputation alone. Concrete mechanisms are required to demonstrate the ethical use of personal data and build consumer confidence.

\subsection{Responding to the adoption challenges}

It might be thought that these challenges will be met through legal measures mandated by GDPR (2016), including data protection by design and default (Article 25), data impact assessment (Article 35), and the information to be provided to data subjects (Articles 13 \& 14) that is typically contained in a service provider's privacy policy. However, our respondents did not appeal to the law as a solution, and this at a time when GDPR's introduction was imminent and widely reported, but instead sought much more tangible means of redress. As noted above, respondents viewed the local use and deletion of data after their visit to LRoTF positively.

Sal: I prefer the idea of the Databox to outside influence or control. I think it's a level of trust. If you're handing over almost your safety, in some senses, to an outside company or person, then you need to be able to trust that they will keep that safety in mind. 
Respondents saw the Databox as a potential means of underwriting their personal safety and abnegating the need to trust 'an outside company or person'. The Databox is a physical device that sits at the edge of the network in the user's home and allows the user to control who can access their data. It exploits a familiar ecosystem of apps that allow external parties to access the user's personal data, and to actuate connected devices, should the user allow it. Importantly the Databox does not let an app take a user's data away for processing; rather, processing of data is done locally, meaning that only the result of a local processing query is distributed. In the context of the LRoTF this means that the interactions that trigger the personalisation of media are not distributed, only the instruction that results from the local processing of that data (e.g., water bottle picked up = X: trigger scene Y).

In radically minimising data distribution the Databox provides a means of addressing many of the adoption challenges confronting adaptive, physically immersive, data-driven experiences: apps cannot be installed in the box without a 'manifest', which describe to the user what an app does, the data it processes and for what purposes, and apps of course have their own interfaces that can enable inspection of data processing and end-user customisation and control (see Crabtree et al. 2018 for further detail). The Databox puts the end-user or media consumer in the driving seat, preserving their autonomy and providing concrete assurance that their privacy is not under threat and that data cannot be used for dystopian purposes, as it remains on-the-box. Thus, in leveraging the Databox, service providers can demonstrate that they are involved in a symmetrical relationship of mutual value and committed to the ethical use of personal data. The adoption challenges identified in this study are not insurmountable then. Indeed our respondents see ways and means by which service providers can build consumer confidence in highly personalised, physically immersive, data-driven experiences. ${ }^{3}$

\section{Conclusion}

Emergent media services are becoming increasingly data-driven in a bid to deliver highly personalised, uniquely customised content. We have presented the design and in-the-wild evaluation of the Living Room of the Future (LRoTF), an innovative installation that exploits object based media and Internet of Things devices to deliver personalised as well as physically immersive data-driven media experiences. The contribution of this paper is two-fold: on the one hand the LRoTF initiates an innovative methodology and generally applicable approach for understanding the adoption challenges confronting future and emerging technologies; and on the other, in it is use, the LRoTF reflexively elaborates substantive adoption challenges confronting the uptake of hyperpersonalised, adaptive, physically immersive, data-driven media experiences in everyday life.

Methodologically, the LRoTF exploits a design fiction approach to merge and prototype future and emerging technologies and situate them in a mundanely familiar setting to provoke critical reflection through the embodied experience of participants. Substantively, we find through in-the-wild deployment a spectrum of adoption challenges confronting the LRoTF including data legibility, potential dystopias, agency and control issues, the social need for customisation, asymmetrical value trade-off, and lack of trust. We also find that these challenges are not insurmountable and that solutions that promote the local processing of data and radical minimisation in data distribution may build widespread consumer confidence in novel media services that rely on the exploitation of personal data. We find too, insofar as the challenges of adoption can be addressed, that hyper-personalised, object-based media that exploit connected Internet of Things devices might deliver mad, intense, magical experiences that immerse viewers in content that reaches out beyond the screen to engage with their physical environment and their physical senses and make the media come alive.

\section{ACKNOWLEDGEMENTS}

This work was supported by the Arts and Humanities Research Council [AH/R008728/1] and the Engineering and Physical Sciences Research Council [EP/M001636/1].

\section{REFERENCES}

BBC (2016) BBC Primer: A Short Summary of BBC R\&D's Work on Near Live Production. https://www.bbc.co.uk/programmes/p03t17gx [Accessed 31 January, 2019].

Bentley, F., Buchner, K. and Kaye, J. (2014.) "MyChannel: exploring city-based multimedia news presentations on the living room TV". Proceedings of the International Conference on Interactive Experiences for TV and Online Video, pp. 71-78, Newcastle Upon Tyne, ACM Press.

\footnotetext{
${ }^{3}$ It was suggested in discussion of this paper that its findings are culturally limited; that the same issues would not be surfaced in the US or Asia, where people are less concerned with what happens to their data. Whether or not this is true is, of course, a matter for empirical investigation, though initial studies with regard to the IoT suggest that, while differences exist, matters are not so clear cut in the US at least (Zheng et al. 2018).
} 
Bernhaupt, R., Obrist, M., Weiss, A., Beck, E. and Tscheligi, M. (2007) “Trends in the living room and beyond." Proceedings of the $5^{\text {th }}$ European Conference on Interactive Televisin, pp. 146-155, Amsterdam, Springer.

British Council (2018) The Living Room of the Future. https://kosovo.britishcouncil.org/en/the-living-room-of-the-future [Accessed 31 January, 2019].

Brown, A., Evans, M., Jay, C., Glancy, M. Jones, R. and Harper, S. (2014) “HCI over multiple screens.” Extended Abstracts on Human Factors in Computing Systems, pp. 665-674, Toronto, ACM Press.

Cesar, P., Bulterman, D.C.A. and Jansen, A.J. (2008) "Usages of the secondary screen in an interactive television environment: control, enrich, share, and transfer television content". Proceedings of the 6th European Conference on Interactive Television, pp. 168-177, Salzburg, Springer.

Cesar, P. and Chorianopoulos, K. (2009) “The evolution of TV systems, content, and users toward interactivity". Foundations and Trends in Human-Computer Interaction, 2(4): 279-373.

Coulton, P. (2015) "Playful and gameful design for the Internet of Things". More Playful Interfaces: Interfaces that Invite Social and Physical Interaction (ed. Nijholt, A.), pp. 151-173, London, Springer.

Coulton, P. (2017) “Sensing atoms and bits”. Sensory Arts and Design (ed. Heywood, I.), pp. 189-204, London, Bloomsbury.

Coulton, P., Lindley, J. and Cooper, R. (2018) The Little Book of Design Fiction for the Internet of Things. https://www.petrashub.org/the-little-book-of-design-fiction-for-the-internet-of-things/ [Accessed 31 January, 2019].

Crabtree, A, Chamberlain, A., Grinter, R.E., Jones, M., Rodden, T. and Rogers, Y. (2013) "Introduction to the special issue of 'The Turn to The Wild'." ACM Transactions on Computer-Human Interaction, 20(3): Article No. 13.

Crabtree, A., Rodden, T., Tolmie, P., Mortier, R., Lodge. T., Brundell, P. and Pantidi, N. (2014) "House rules: the collaborative nature of policy in domestic networks", Personal and Ubiquitous Computing, 19(1): 203-215.

Crabtree, A., Lodge, T., Colley, J., Greenhalgh, C., Glover, K., Haddadi, H., Amar. Y., Mortier, R., Li, Q., Moore, J., Wang, L., Yadav, P., Zhao, J., Brown, A., Urquhart, L. and McAuley, D. (2018) "Building accountability into the internet of things: the IoT Databox model". Journal of Reliable Intelligent Environments, 4(1): 39-55.

GDPR (2016) Regulation 2016/679 General Data Protection Regulation. Official Journal of the European Union, vol. 59, pp. 1-149.

Gradinar, A., Burnett, D., Coulton, P., Forrester, I., Watkins, M., Scutt, T. and Murphy, E. (2015) “Perceptive Media adaptive storytelling for digital broadcast". Proceedings of the IFIP Conference on Human-Computer Interaction, pp. 586-589, Bamberg, Springer.

Hanley, P. and Viney R. (2009) "Pressing the red button: consumers and digital television.” Cultural Trends, 11(43-44): 35-60.

Hook, J. (2018) "Facts, interactivity and videotape: exploring the design space of data in interactive video storytelling." Proceedings of the ACM TVX International Conference on Interactive Experiences for Television and Online Video, pp. 43-55, Seoul. ACM Press.

Huang, Y.C., Wu, K.Y. and Liu, Y.T. (2013) "Future home design: an emotional communication channel approach to smart space.” Personal and Ubiquitous Computing, 17(6): 1281-1293.

Kerlin, L., Cox, J., Jolly, S., Evans. M., Green, G. and Regan, D. (2016) "Pressing not tapping: comparing a physical button with a smartphone app for tagging music in radio programmes." Proceedings of the SIGCHI Conference on Human Factors in Computing Systems, pp. 5874-5884, San Jose, ACM Press.

Langheinrich, M. (2002) “A privacy awareness system for ubiquitous computing environments.” Proceedings of the $4^{\text {th }}$ International Conference on Ubiquitous Computing, pp. 237-245, Gothenberg, Springer.

Lanier, J. (2014) Who Owns the Future? New York, Simon and Schuster.

Lindley, J., Coulton, P. and Sturdee, M. (2017) “Implications for adoption”. Proceedings of the SIGCHI Conference on Human Factors in Computing Systems, pp. 265-277, Denver, ACM Press.

McAlone, N. (2015) "Netflix has created 'smart' socks that sense when you fall asleep and pause the show you're watching.” Business Insider, http://uk.businessinsider.com/netflix-socks-turn-off-the-show-when-you-fall-asleep2015-12/\#first-you-assemble-the-components-1 [Accessed 31 January, 2019]. 
Mortier, R., Haddadi, H., Henderson, T., McAuley, D., Crowcroft, J. and Crabtree, A. (2016) "Human Data Interaction”. Encyclopedia of Human-Computer Interaction, Chapter 41, Interaction Design Foundation.

Moulding, J. (2017) TVlights is the best example yet of TV/IoT integration, and a realistic near-term immersive TV experience." Videonet, https://www.v-net.tv/2017/07/21/tvlights-is-the-best-example-yet-of-tviot-integration-anda-realistic-near-term-immersive-tv-experience/ [Accessed 31 January, 2019].

Obrist, M., Cesar, P., Geerts, D., Bartindale, T. and Churchill, E. (2015) “Online video and interactive TV experiences”. ACM Interactions Magazine, 22(5): 32-37.

Pollner, M. (2010) Mundane Reason: Reality in Everyday and Sociological Discourse. Cambridge, Cambridge University Press.

Regan, C. (1995) “An investigation into nausea and other side-effects of head-coupled immersive virtual reality”. Virtual Reality, 1(1): 17-31.

Rogers, Y. and Marshall, P. (2017) Research in the Wild. http://www.morganclaypool.com/doi/10.2200/S00764ED1V01Y201703HCI037 [Accessed 31 January, 2019].

Rose, J., Rehse, O. \& Röber, B (2012) “The value of our digital identity.” BCG, https:/www.bcg.com/publications/2012/digital-economy-consumer-insight-value-of-our-digital-identity.aspx [Accessed 31 January, 2019].

Sailaja, N., Crabtree, A., McAuley, D. and Stenton, P. (2018) "Explicating the challenges of providing novel media experiences driven by user personal data." Proceedings of the International Conference on Interactive Experiences for TV and Online Video, pp. 101-113, Seoul, ACM.

Sailaja, N., Crabtree, A. and Stenton, P. (2017) "Challenges of using personal data to drive personalised electronic programme guides.” Proceedings of the SIGCHI Conference on Human Factors in Computing Systems, pp. 52265231, Denver, ACM Press.

Schofield, G., Bartindale, T. and Wright, P. (2015) "Bootlegger: turning fans into film crew". Proceedings of the ACM SIGCHI Conference on Human Factors in Computing Systems, pp. 767-776, Seoul, ACM Press.

Shotton, M., Melchior, F., Evans, M., Churnside, T, Brooks, M and Armstrong, M. (2015) “Object-based Broadcasting curation, responsiveness and user experience". White Paper WHP 285, BBC R\&D.

http://downloads.bbc.co.uk/rd/pubs/whp/whp-pdf-files/WHP285.pdf [Accessed 31 January, 2019].

Smith, D. (2018) Redefining Television through Object-Based Broadcasting. https://www.digitalcreativity.ac.uk/projects/redefining-television-through-object-based-broadcasting [Accessed 31 January, 2019].

Ursu, M., Kegel, I.C., Williams, D., Thomas, M., Mayer, H., Zsombori, V., Tuomola, M.L., Larsson, H. and Wyver, J. (2008a) "ShapeShifting TV: interactive screen media narratives.” Multimedia Systems, 14(2): 115-132.

Ursu, M., Thomas, M., Kegel, I., Williams, D., Tuomola, M., Lindstedt, I., Wright, T., Leurdijk, A., Zsombori, V., Sussner, J., Myrestam, U. and Hall, N. (2008b) Interactive TV narratives: opportunities, progress, and challenges". ACM Transactions on Multimedia Computing, Communications, and Applications, 4(4): Article No. 25.

WEF (2011) "Personal data: the emergence of a new asset class". World Economic Forum, http://www3.weforum.org/docs/WEF_ITTC_PersonalDataNewAsset_Report_2011.pdf [Accessed 31 January, 2019].

WEF (2014) "Rethinking personal data: a new lens for strengthening trust." World Economic Forum, http://www3.weforum.org/docs/WEF_RethinkingPersonalData_ANewLens_Report_2014.pdf [Accessed 31 January, 2019].

Zheng, S., Apthorpe, N., Chetty, M. and Feamster, N. (2018) "User perceptions of smart home IoT privacy." Proceedings of the ACM on Human-Computer Interaction-CSCW, 2: Article 200. 